\title{
Relation of daily periods of vlf radiation with X-ray sources
}

\author{
Gennady Drugin ${ }^{1, \star}$ and Ivan Stasiy ${ }^{1}$ \\ ${ }^{1}$ Institute of Cosmophysical Researches and Radio Wave Propagation FEB RAS, \\ Paratunka, Kamchatsky Kray, Russia
}

\begin{abstract}
Spectral analysis of electromagnetic noise radiation in the VLF range at three fixed frequencies for the period from 1997 to 2015 have been carried out. Periodograms with diurnal components associated with the periods of the Earth rotation relatively the Sun and the stars have been obtained. It has been shown that the Sun x-radiation and the galaxy x-radiation affect daily variations of VLF radiation.
\end{abstract}

\section{Introduction}

The Sun x-radiation is the major factor affecting the ionosphere D region http://ru.wikipedia.org/, $[1,2]$. Additional weak sources of ionization such as meteorites burning up in the atmosphere at the heights of 60-100 km, cosmic rays, energetic particles of the magnetosphere brought into this layer during magnetic storms also have some impact on the $\mathrm{D}$ region ionization process which mainly affect the conditions of radio wave propagation in a very low frequency range.

Sun daily rotation ( $\sim 27$ days) as well as the week cycle of human activity may also manifest themselves in the spectra of electromagnetic VLF radiation received on the Earth [3]. However, there are only single cases when daily periods of VLF radiation are under consideration $[4,5]$.

The aim of the paper is the investigation of noise characteristics of electromagnetic VLF signals in the period ranges close to the Earth daily rotation on large time intervals and determination of their potential relation with $\mathrm{x}$-radiation sources.

In order to do that, we have carried out spectral analysis of VLF radiation noise envelope at three fixed frequencies based on the data of long-term registration in the period range close to the Earth daily rotation.

\section{Instrumentation and methods of investigation}

Electromagnetic noise radiation was registered by a multichannel VLF-detector located in Kamchatka at «Karymshina» field site $\left(\varphi=52^{\circ} 49^{\prime} \mathrm{N}, \lambda=158^{\circ} 07^{\prime} \mathrm{E}\right)$ The VLF radiation envelope is recorded at three fixed frequencies in narrow frequency bands (5\% - 10\% from the band center). The VLFdetector consists of a loop antenna, pre-amplifier, communication cable, signal filtration unit, output device. The loop antenna has a size of 7,5x15 m and its plane is oriented in the east-western direction.

Spectral analysis was carried out for the radiation envelope at the frequencies of $0.7,1.2,5.3 \mathrm{kHz}$ near the daily periods. Fig. 1, a shows a periodogram for the registration period from 1997 to 2006

^e-mail: drug@ikir.ru 
(10 years). It is clear from the figure that there is the main maximum at the period of $1440 \mathrm{~min}$ ( 1 day) at all the frequencies. This period is associated with the Earth daily rotation relatively the Sun. Moreover, another maximum less in value is observed at the period of $1436 \mathrm{~min}$. This period coincides with the period of Earth rotation relatively remote stars. Periodograms for the registration period indicated above were illustrated in the paper $[4,5]$.
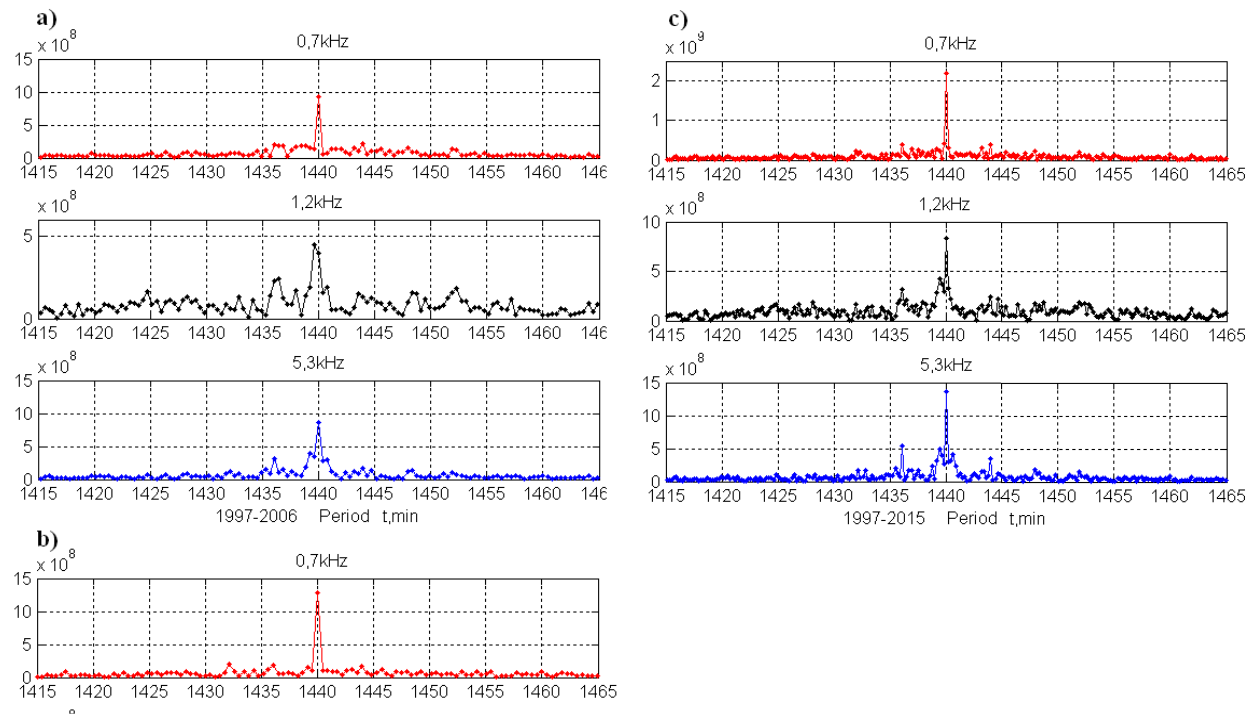

1997-2015 Period t,min
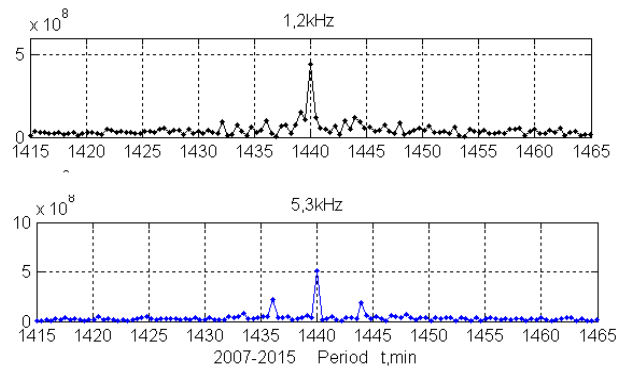

Figure 1. eriodograms of VLF noise envelope at three frequencies near daily periods based on observations for a) $1997-2006$ b) $2007-2015$ c) $1997-2015$.

Further analysis of the radiation envelope near the daily periods at the same frequencies was carried out for the period from 2007 to 2015 (9 years). It is clear from Fig. 1,b that there are main and additional maxima at the periods of 1440 and 1436 min during the observation period. There is another maximum at the period of 1444 min which is more distinct at the frequency of $5.3 \mathrm{kHz}$. This maximum does not coincide with daily periods of the Earth rotation relatively the Sun and the stars. It is most likely to be determined by the annual rotation of the Earth around the Sun since the daily variation of the VLF radiation envelope at the frequency $f_{0}$ is modulated by the annual variation with the frequency $\Delta f$. Thus, due to the annual variation, two components similar in amplitude at 
the frequencies of $f_{1}=f_{0}+\Delta f$ and $f_{2}=f_{0}-\Delta f$, ( $\Delta f$ is the modulation frequency)should appear in a signal spectrum besides the fundamental frequency of $f_{0}$. In our case, the fundamental period is $T_{0}=1 / f_{0}=1440 \mathrm{~min}, T_{1}=1 / f_{1}=1436 \mathrm{~min}, T_{2}=1 / f_{2}=1444 \mathrm{~min}$. Only the period $T_{1}$ coincides with the «star» one out of these «annual» periods.

Fig. 1,c shows a periodogram for the registration period from 1997 to 2015 (19 years). The merged data sets (1997 - 2006 and 2007 - 2015) for spectral analysis allowed us to improve the oscillatory period resolution. On this periodogram, maxima at the periods of 1440 and $1436 \mathrm{~min}$ are more distinguished. There are maxima at the periods of 1444 min but they are less in amplitude.

Now we consider the dependence of the detected maxima on observation periods, noise and registration frequency. We specify the natural noise radiation as a signal, and the instrumentation selfnoises and different other kinds of disturbances as noise. The average of noise is calculated for the spectral component range from 1415 to 1465 min excluding the period from 1435 to 1445 within which the signals associated with daily and annual variation of natural electromagnetic radiation. Then signal amplitude values at the periods of $T_{1}, T_{0}$ and $T_{2}$ are divided by the noise average and the results are written into the table.

Tables $1-3$ show spectral component $\mathrm{K}$ values divided by the noise averages for different time intervals of VLF radiation for the envelopes at three frequencies.

Table 1

\begin{tabular}{|l|l|l|l|}
\hline \multicolumn{4}{|c|}{$1997-2006$} \\
\hline $\mathrm{T}(\mathrm{min})$ & 1436 & 1440 & 1444 \\
\hline $\mathrm{K}(0.7 \mathrm{kHz})$ & 4.5 & 20.8 & 4.6 \\
\hline $\mathrm{K}(1.2 \mathrm{kHz})$ & 3.2 & 5.7 & 1.8 \\
\hline $\mathrm{K}(5.3 \mathrm{kHz})$ & 7.9 & 21.4 & 4.2 \\
\hline
\end{tabular}

Table 2

\begin{tabular}{|l|l|l|l|}
\hline \multicolumn{4}{|c|}{$2007-2015$} \\
\hline $\mathrm{T}(\mathrm{min})$ & 1436 & 1440 & 1444 \\
\hline $\mathrm{K}(0.7 \mathrm{kHz})$ & 3.9 & 26.9 & 3.6 \\
\hline $\mathrm{K}(1.2 \mathrm{kHz})$ & 3.1 & 13.6 & 3.6 \\
\hline $\mathrm{K}(5.3 \mathrm{kHz})$ & 9.2 & 21.0 & 4.9 \\
\hline
\end{tabular}

Table 3

\begin{tabular}{|l|l|l|l|}
\hline \multicolumn{4}{|c|}{$1997-2015$} \\
\hline $\mathrm{T}(\mathrm{min})$ & 1436 & 1440 & 1444 \\
\hline $\mathrm{K}(0.7 \mathrm{kHz})$ & 5.8 & 32.7 & 5.6 \\
\hline $\mathrm{K}(1.2 \mathrm{kHz})$ & 4.0 & 10.7 & 3.1 \\
\hline $\mathrm{K}(5.3 \mathrm{kHz})$ & 11.4 & 28.7 & 7.3 \\
\hline
\end{tabular}

It is clear from Tables $1-3$ that $\mathrm{K}$ main daily maximum at the period of $T_{0}=1440 \mathrm{~min}$ significantly exceeds the noise level as well as $\mathrm{K}$ maxima at the periods of $T_{1}=1436 \mathrm{~min}$ and $T_{2}=1444$ $\mathrm{min}$ at all the registered frequencies. For the most part, $\mathrm{K}$ maxima at the periods of $1436 \mathrm{~min}$ and 1444 min also significantly exceed the noise level.

For the observation period of 1997-2006 (Table 1), K values at the frequency of $0.7 \mathrm{kHz}$ at the periods of $1436 \mathrm{~min}$ and $1444 \mathrm{~min}$ are almost the same and exceed the noise level by more than 4 times. It proves that at this frequency the K maxima at the periods of $1436 \mathrm{~min}$ and $1444 \mathrm{~min}$ are generally determined by radiation annual variation, and a small difference in the values is due to the noise effect.

At the frequency of $1.2 \mathrm{kHz}$, the difference between $\mathrm{K}$ at the periods of $1436 \mathrm{~min}$ and $1444 \mathrm{~min}$ (Table 1) is more than that considered above that indicates the presence of an additional "star" type source of radiation besides the annual one. We should note that at this frequency the $\mathrm{K}$ values are less than at other frequencies that means that the relative impact of noise is higher. 
At the frequency of $5.3 \mathrm{kHz}$, the $\mathrm{K}$ values are the highest for all the periods $\mathrm{T}$ shown in Table 1 . Both "star" and "annual" periods are clearly distinguished at this frequency.

Almost everything that has been described for the observation period from 1997 to 2006 is true for the period from 2007 to 2015 excepting that at the frequency of $1.2 \mathrm{kHz}$ the $\mathrm{K}$ values at the periods of $1436 \mathrm{~min}$ and $1444 \mathrm{~min}$ are almost similar. "Annual" period appears at this frequency and a small exceedance of $\mathrm{K}$ at the period of $1444 \mathrm{~min}$ over the period of $1436 \mathrm{~min}$ is explained by noise effect.

$\mathrm{K}$ values for the whole period of observations from 1997 to 2015 (Table 3) show that with observation time increment, the signal/noise relation increases, and "star" period is distinguished more clearly. "Annual" periods are also distinctly observed, for example, at the frequency of $0.7 \mathrm{kHz}$ where the difference between $\mathrm{K}$ values at the periods of $1436 \mathrm{~min}$ and $1444 \mathrm{~min}$ is small. Just like in the previous cases, "star" period of $1436 \mathrm{~min}$ at the frequency of $5.3 \mathrm{kHz}$ is clearly observed.

\section{Sources of radiation}

It is known that the main sources of natural electromagnetic radiation in VLF range are lightning strokes. When such radiation is received, the level of a signal depends on the intensity of a lightning source and the conditions of signal propagation along the path. In summer time, radiation from nearby (regional) lightning sources is mainly registered in the North-East of Russia. During this period, the level of the received noise is maximal. In winter time, there is no local lightning, and the VLF receiver registers remote lightning located in the near equatorial regions for the most part. Radiation from the world lightning centers is recorded [6]. During this time, the level of the signal depends significantly on the conditions of signal propagation along the path. At nighttime radiation generated by the sources at the distances of $10000 \mathrm{~km}$ from the registration site is registered.

When radiation propagates from remote sources, the main factor affecting registration of VLF signals is the Sun x-radiation. In the paper [1] the authors found a close relation of VLF noise intensity variations with $\mathrm{x}$-radiation flux changes during solar flares. Whereas, the paper [2] considers the method of estimation of x-radiation fluxes from solar flares by the level of absorption of regular noise VLF radiation. The intensity of solar flare x-radiation may reach high values. For example, the maximum intensity of a flare which occurred on November 4, 2004 was $28 \cdot 10^{-4} \mathrm{~W} / \mathrm{m}^{2}$ [http://ru.wikipedia.org/wiki/]. The capability of x-ray flares to cause sudden ionospheric disturbances depends both on the flux value and on the degree of stiffening during a flare estimated as a relation of flux levels in spectral intervals $0-3 A^{0}$ and $0-8 A^{0}$ [7]. Besides the solar radiation, there is also galactic radiation in the $\mathrm{x}$-radiation range which may also influence the level of the registered VLF noise affecting the D and E regions of the ionosphere. S.L. Mandelshtam wrote [http://wrabbit.narod.ru/raznoe/rentgen.htm], hat by the Earth's atmosphere boundary, the Sun X-radiation flux in the region of $1-10$ angstrom is $10^{-4} \mathrm{erg} /\left(\mathrm{sm}^{2} \mathrm{~s}\right.$ ) (total flux of electromagnetic radiation from the Sun by the Earth's atmosphere boundary is $1.5 \cdot 10^{6} \mathrm{erg} /\left(\mathrm{sm}^{2} \mathrm{~s}\right)$, and the most powerful source of the Galaxy is in Scorpio constellation Sco X-1, the flux from which is $5 \cdot 10^{-7} \mathrm{erg} /\left(\mathrm{sm}^{2} \mathrm{~s}\right.$ ) (from the galaxy, $10^{-4}-10^{-3}$ of optical luminosity fall on the $\mathrm{x}$-ray range). There are about a hundred of discrete x-ray sources. The x-radiation is also observed from the Galactic ridge x-ray emission. It is extensive radiation of low surface brightness arranged as a band with the width of about 1 2 degrees along the Galaxy plane [http://ru.wikipedia.org/wiki/]. Moreover, based on reasonable, statistically valid estimates, we can expect about a hundred of metagalactic x-ray sources [http://wrabbit.narod.ru/raznoe/rentgen.htm], but the flux from these sources coming to the Earth's atmosphere is small due to the great distance. It follows from the above that the x-radiation flux from the Galaxy differs in value from that from the Sun by the Earth's atmosphere boundary. It also may affect the level of ionization of the ionosphere $\mathrm{D}$ and $\mathrm{E}$ regions that causes the change of the registered VLF radiation amplitude. 
Besides the $\mathrm{x}$-radiation, ionospheric and magnetospheric storms as well as industrial noises, circuit noises of $50 \mathrm{~Hz}$ and its harmonics may affect registration of noise VLF signals. Ionosphericmagnetospheric storms make the major impact during the registration of VLF signals at high latitudes, in the auroral belt. Industrial noises are mainly observed near towns and manufacturing areas. In our case, the observation site is located far from the auroral belt and from noises of industrial origin. However, there is some small impact of the mentioned sources on the noise component of VLF signals.

$\mathrm{X}$-radiation influencing the process of ionosphere ionization affects the frequency

characteristics of the registered VLF signals in different ways. It was noticed that the radiation noise level usually increased in the frequency range less than $1 \mathrm{kHz}$ during solar $\mathrm{x}$-ray flares, whereas it significantly decreased at the frequencies of $4-5 \mathrm{kHz}$. In our case, the registered radiation at $0.7 \mathrm{kHz}$ and $1.2 \mathrm{kHz}$ is in the transition frequency region which is weakly affected by the x-radiation. Thus, the impact of $\mathrm{x}$-ray sources at these frequencies is minimal. Almost similar $\mathrm{K}$ values at the periods of $1436 \mathrm{~min}$ and $1444 \mathrm{~min}$ at these frequencies (Tables 1 - 3) indicate that "annual" impact is greater than the "star" one.

The frequency of $5.3 \mathrm{kHz}$ falls within the range with maximum influence of $\mathrm{x}$-radiation, thus, the effect should be more significant at this frequency that is observed in K values (Tables $1-3$ ). Considerable exceedance of $\mathrm{K}$ at the period of 1436 in comparison to the period of 1444 shows that the "star" source prevails over the "annual" one.

\section{Conclusions}

We have carried out a spectral analysis of noise electromagnetic radiation envelope at the frequencies of $0.7,1.2,5.3 \mathrm{kHz}$ near the daily periods during the observations from 1997 to 2006 (10 years), from 2006 to 2015 (9 years) and for the whole period of observations from 1997 to 2015 (19 years).

Radiation maxima are observed in spectral components at the periods of 1436, 1440 and 1444 min. All the recorded maxima for 1997-2015 exceed the average noise by more than 3 times. The maximum exceedance of the radiation over noise is 28.7 , and it is observed at the period of $1440 \mathrm{~min}$ for the received frequency of $5.3 \mathrm{kHz}$.

The spectral component at the period of $1440 \mathrm{~min}$ is determined by the Earth daily rotation relatively the Sun, whereas at the period of 1436 it is determined by the Earth daily rotation relatively the stars. There are also annual variations at the periods of 1436 and $1444 \mathrm{~min}$ in the spectral components of noise electromagnetic radiation.

It has been shown that the Galaxy x-radiation may affect the ionization level of ionosphere D region and its influence causes a maximum in noise VLF radiation spectrum at the period of 1436 min associated with the Earth rotation relatively the stars. The obtained result may promote better understanding of the processes affecting the $\mathrm{D}$ region of the ionosphere.

\section{References}

[1] N. N. Murzaeva, Relation of VLF radiation of the upper atmosphere with other geophysical phenomena (YB SB AS USSR, Yakutsk, 1977), 124

[2] V. A. Mullayarov, R. R. Karimov, V. I. Kozlov, N. N. Murzaeva, Meteorology and Hydrology, 8, 48-56 (1998)

[3] V. I. Kozlov, V. A. Mullayarov, Lightning activity in Yakutiya (YB SB RAS, Yakutsk, 2004), 104

[4] G. I. Druzhin, A. N. Mel'nikov, N. V. Cherneva, Doklady Earth Sciences, 457:1, 92-94 (2014)

[5] G. I. Druzhin, International research journal, 8:39, 98-101 (2015) 
[6] G. I. Druzhin, V. I. Shapaev, Geomagnetism and Aeronomy, 28:1, 81-86 (1988)

[7] A. Mitra, Effects of solar flares on Earth's ionosphere (Mir, Moscow, 1977), 370 